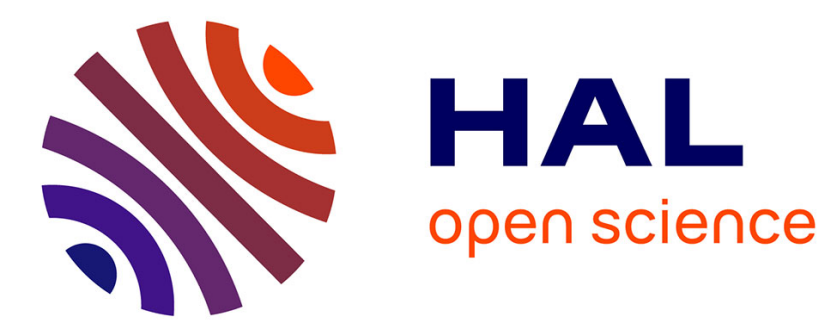

\title{
Drop fragmentation due to hole formation during Leidenfrost impact
}

\author{
Anne-Laure Biance, Christophe Pirat, Christophe Ybert
}

\section{To cite this version:}

Anne-Laure Biance, Christophe Pirat, Christophe Ybert. Drop fragmentation due to hole formation during Leidenfrost impact. Physics of Fluids, 2011, 23 (2), 10.1063/1.3553277 . hal-01628774

\section{HAL Id: hal-01628774 \\ https://hal.science/hal-01628774}

Submitted on 3 Dec 2017

HAL is a multi-disciplinary open access archive for the deposit and dissemination of scientific research documents, whether they are published or not. The documents may come from teaching and research institutions in France or abroad, or from public or private research centers.
L'archive ouverte pluridisciplinaire HAL, est destinée au dépôt et à la diffusion de documents scientifiques de niveau recherche, publiés ou non, émanant des établissements d'enseignement et de recherche français ou étrangers, des laboratoires publics ou privés. 


\title{
Drop fragmentation due to hole formation during Leidenfrost impact
}

\author{
Anne-Laure Biance, ${ }^{\text {a) }}$ Christophe Pirat, ${ }^{\text {b) }}$ and Christophe Ybert $^{\mathrm{c})}$ \\ Laboratoire de Physique de la Matière Condensée et Nanostructures (LPMCN), UMR 5586 CNRS and \\ Université de Lyon; Univ. Lyon I, 69622 Villeurbanne, France
}

(Received 13 July 2010; accepted 21 December 2010; published online 11 February 2011)

\begin{abstract}
Drop impacts on a smooth plate heated above the Leidenfrost temperature are investigated in the range of large Weber number. Liquid fragmentation due to the rupture of the expanding lamella during the impact—by hole nucleation and subsequent growth—is studied. Control of this rupturing process is achieved experimentally through the use of single model-defects attached to the substrate which act as an initiating spots for the hole formation, whereas the liquid does not contact the substrate. Overall, the lamella rupture is shown to take place above a critical impact velocity, the value of which decreases with increasing defect size. Comparing this rupture mechanism to classical splash, it is shown to be the relevant fragmentation phenomenon below a critical ratio between drop $\left(R_{0}\right)$ and defect $(d)$ sizes of $R_{0} / d \leq 40$. (C) 2011 American Institute of Physics.

[doi:10.1063/1.3553277]
\end{abstract}

Drop impact onto solid surfaces is a common physical phenomenon that takes place in a wide range of practical situations as inkjet printing, ${ }^{1}$ pesticide delivery, ${ }^{2}$ and various spray coatings. The particular case of impact on hot plates has been extensively studied, as it is a major issue in many cooling and transfer processes involved in fuel combustion or spray cooling. From a more fundamental point of view, the physics of impact is very rich and despite more than a century of studies initiated by Worthington, ${ }^{3}$ many issues remain unsolved. When a drop impacts on a solid surface, it can spread, stick, or bounce depending on the control parameters that include surface roughness, wettability, and liquid properties (surface tension, density, and viscosity). We focus here on the case of bouncing, provided that the material is highly hydrophobic. As a model situation, Leidenfrost impacts on a hot solid plate are considered where a thin vapor layer suspends the drop, preventing any contact between the liquid and the substrate. Typically, such suspending vapor layer is about $60 \mu \mathrm{m}$ thick for millimetric water drops at rest $^{4}$ and thinner than $10 \mu \mathrm{m}$ in dynamical situation. ${ }^{5}$

An important concern in the physics of impact is the formation of satellite-so-called splash-droplets, which result from the destabilization of the edge of the spreading drop. The relevant parameters controlling splash are still a field of active research: ${ }^{6,7}$ ambient atmosphere, ${ }^{8}$ surface wettability, ${ }^{7}$ surface defects, ${ }^{9}$ structuration, ${ }^{10}$ and compliance, ${ }^{11}$ being all key factors determining splash behavior.

Concurrent to the above splash mechanism, here we investigate another phenomenon resulting in droplet formation, which is the consequence of hole nucleation in the radially spreading liquid film. Formation of such holes has already been observed in different wetting situations, ${ }^{12-15}$ where it

\footnotetext{
a) Electronic mail: anne-laure.biance@univ-lyon1.fr.

${ }^{b)}$ Electronic mail: christophe.pirat@univ-lyon1.fr.

${ }^{\text {c) }}$ Electronic mail: christophe.ybert@univ-lyon1.fr.
}

has been attributed to bubble entrapment during impact or to thermodynamic instability. ${ }^{16}$ Moreover, here we show that such hole formation is very sensitive to the presence of single microsized defects that can be used to trigger and control it. We study the relevance of this hole-opening mechanism for drop fragmentation - as compared to classical splash. The article is organized as follows. First, the conditions of hole formation on a smooth surface are characterized. Then the role of single model-defects with controlled size on these formation conditions is investigated, and a model is proposed that predicts when hole formation occurs. Eventually, the relevancy of the subsequent breakup mechanism compared to classical splash is discussed.

In the experiments, a liquid drop of radius $R_{0}$ varied between 0.7 and $2 \mathrm{~mm}$ is released from a needle at a controlled height (between 2 and $100 \mathrm{~cm}$ ) on a hot silicon wafer above the Leidenfrost temperature. Immediately after reaching the plate, a vapor film is formed between the substrate and the drop bottom surface that reaches the boiling temperature. Furthermore, we assume the whole drop to be at boiling temperature at which the different liquid properties are interpolated from available data. ${ }^{17}$ Two liquids are used (acetone and isopropanol) whose characteristics are presented in Table I. The plate temperature before impact is set to $325^{\circ} \mathrm{C}$ except for a few experiments performed with a plate temperature of $280{ }^{\circ} \mathrm{C}$. No significant effects of temperature are observed in this case. Images are recorded with a high speed video camera up to $13000 \mathrm{frames} / \mathrm{s}$. In these experimental conditions, the Reynolds number is always larger than 300 . Consequently, the impact dynamics is governed by a balance between surface tension and inertia, characterized by the Weber number

$$
\mathrm{We}=\frac{\rho V^{2} R_{0}}{\gamma}
$$

with $\rho$ the liquid density and $\gamma$ the surface tension. 
TABLE I. Properties of the liquid employed during experiments. Boiling point (bp) and Leidenfrost point (Lp) are given in ${ }^{\circ} \mathrm{C}$. Surface tension $\gamma$ and density $\rho$ values are evaluated at the boiling point.

\begin{tabular}{lllcc}
\hline \hline & & & \multicolumn{2}{c}{} \\
& $\mathrm{bp}$ & $\mathrm{Lp}$ & $\begin{array}{c}\gamma \\
\left(\mathrm{kg} / \mathrm{m}^{3}\right)\end{array}$ & $(\mathrm{mN} / \mathrm{m})$ \\
\hline Isopropanol & 82.5 & 165 & 710 & 16 \\
Acetone & 56 & 140 & 750 & 21.2 \\
\hline \hline
\end{tabular}

Depending on the drop radius and velocity, different behaviors of drop impact can be observed. When We is greater than 1 and lower than a critical value for which splashing occurs, the drop spreads due to inertia, recoils and bounces because of surface tension, as depicted in Fig. 1(a). The features (elasticity and maximal deformation) of such a bouncing process on hot plates have been extensively studied in the past. ${ }^{14,18}$ The drop reaches the shape of a thin lamella surrounded by a rim, as observed and modeled in the past 20 years. ${ }^{16,19,20}$

At higher We, satellite droplets are observed during the impact due to the destabilization of the moving edge. This splash mechanism has been studied in many situations. For inertial Leidenfrost impact, the splash threshold is set by a critical Weber number $\mathrm{We}_{c}=160$, as reported in Refs. 21-23 for film boiling. Our experiments are in good agreement with $\mathrm{We}_{c}=150-180$.

If the impact velocity is further increased, another mechanism that can also result in drop fragmentation appears, namely, the formation of a hole in the lamella, as depicted in Fig. 1(c). During spreading, the liquid sheet destabilizes and dewetting is observed. The receding outer rim and the expanding hole front then collide, resulting in the formation of a torus upward, which destabilizes in many satellite droplets, as depicted in Fig. 1(c). Our present purpose is to further characterize this hole forming and expanding phenomenon so as to predict in which conditions it proves relevant for drop fragmentation, as compared to splash.

To achieve such a goal, we first concentrate on the conditions for hole formation by comparing the timescales involved both in the impact process and in the hole formation. Concerning the characteristic timescale associated with the impact process, it is expected to be set, for a bouncing drop on a smooth substrate, by a balance between surface tension and inertia. ${ }^{21,24}$ Consequently, we anticipate it to scale as the oscillation period $\tau_{\text {osc }}$ of the impacting drop in air, where $\tau_{\text {osc }}$ reads

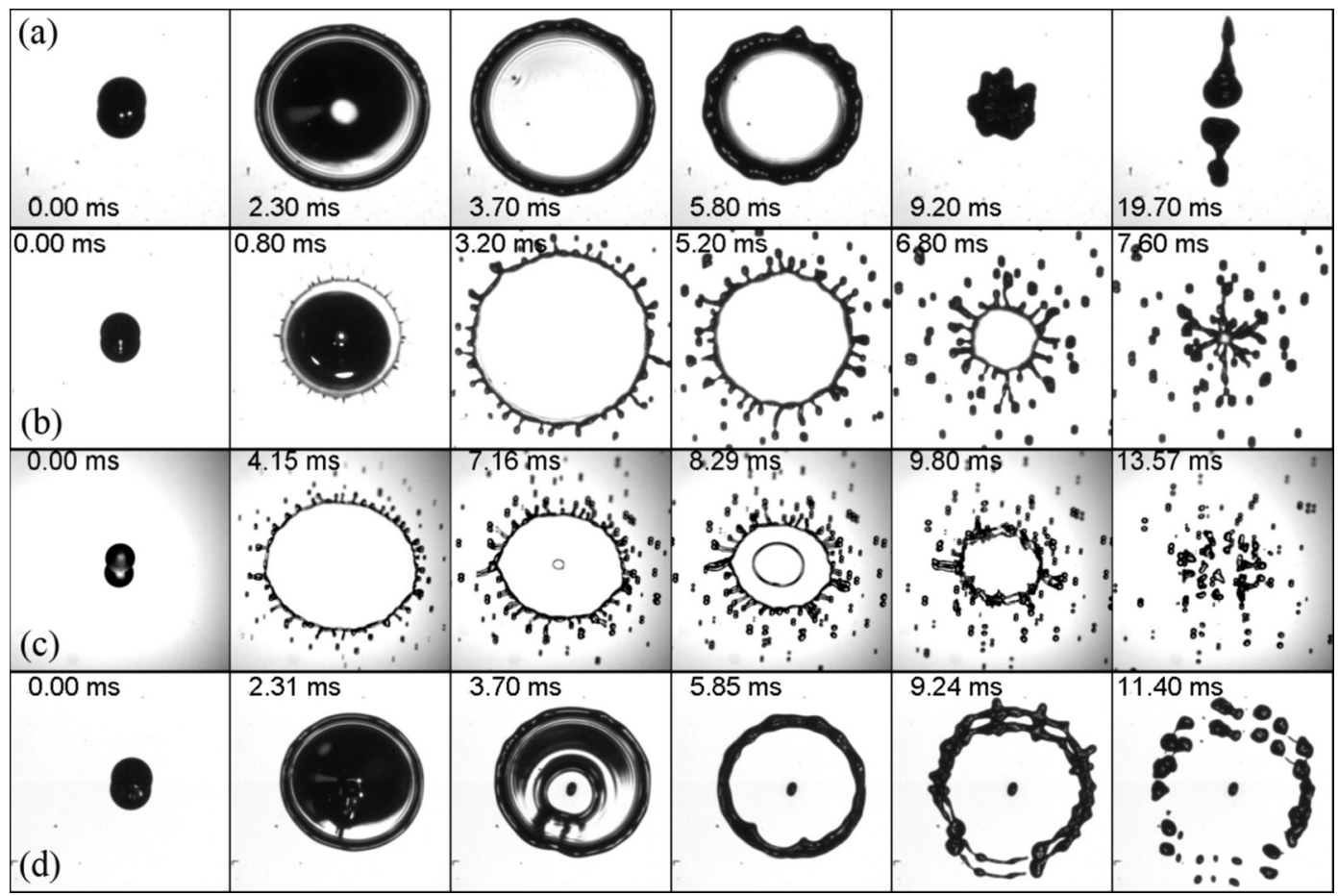

(e)

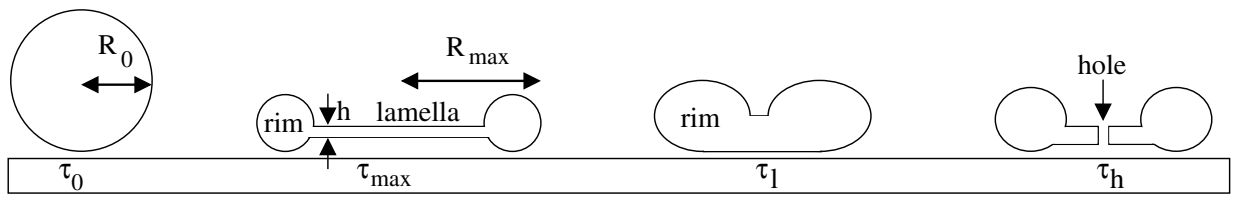

FIG. 1. Image sequence of the impact of isopropanol drop on a silicon wafer. (a) Complete rebound on a smooth substrate $R_{0}=1 \mathrm{~mm}$ and We $=170$. (b) Splash on a smooth substrate at $R_{0}=1 \mathrm{~mm}$ and We $=260$. (c) Splash and hole formation at the center of impact on a smooth substrate at $R_{0}$ $=1.3 \mathrm{~mm}$ and $\mathrm{We}=390$. (d) Hole formation without splash on a defect of size $d=400 \mu \mathrm{m}$ sintered on the substrate, $R_{0}=1 \mathrm{~mm}$ and We=170. (e) Cross section scheme of the impact and hole formation processes. 


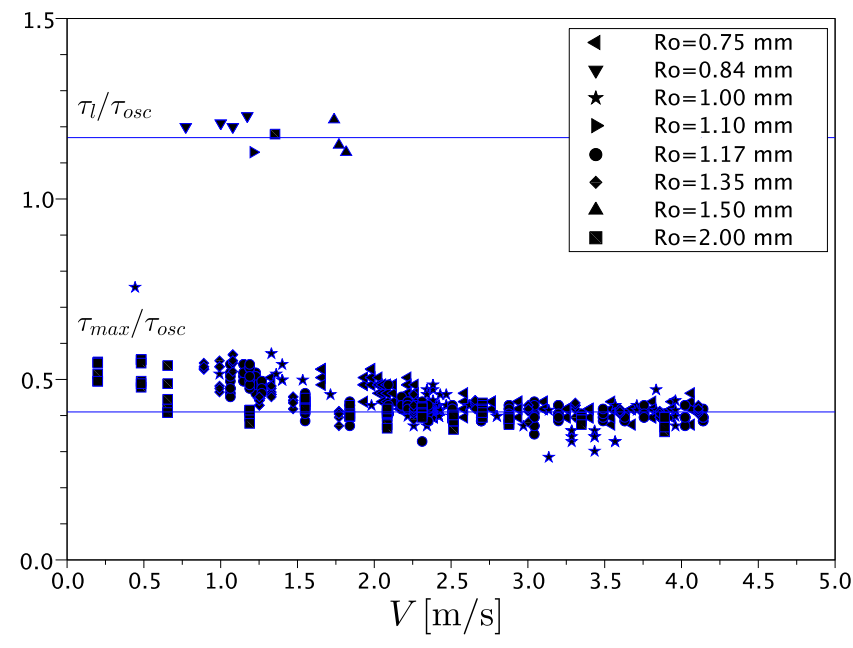

FIG. 2. (Color online) Impact on a smooth substrate. Time to reach the maximal diameter $\tau_{\max }$ and lifetime of the lamella $\tau_{l}$ normalized by $\tau_{\mathrm{osc}} \mathrm{vs}$ impact velocity for several drop radii.

$$
\tau_{\mathrm{osc}}=\sqrt{\frac{\rho R_{0}^{3}}{\gamma}} .
$$

Experimentally, the time $\tau_{\max }$ to reach the maximum diameter after contact provides one such typical time associated with the drop impact. Figure 2 reports the measured $\tau_{\max }$, scaled by $\tau_{\text {osc }}$, for different drop sizes and velocities. In line with the above analysis, this spreading time $\tau_{\max }$ indeed scales essentially as $\tau_{\text {osc }}$, with $\tau_{\text {max }} / \tau_{\text {osc }} \approx 0.41$ on a wide range of impact velocities. Note, however, the slight deviation from this behavior observed at low velocity, similar to what was previously described experimentally ${ }^{25}$ for contact time. These results are in good agreement with observations on superhydrophobic substrate as discussed in Ref. 18, suggesting no important effect of dynamic surface tension.

Parallel to the spreading time, the lamella lifetime $\tau_{l}$, the time for the lamella to close on itself after spreading and recoil [see Fig. 1(e)], yields another natural way to quantify the typical time associated with the impact. Practically, $\tau_{l}$ can be measured as far as the lamella exists (high enough impact velocity) and that no hole is formed, thus restricting the accessible parameter range. Measured lifetime $\tau_{l}$ is reported in Fig. 2, where it appears independent of the impact velocity in agreement with the expected scaling by $\tau_{\text {osc }}$. Overall, the lifetime simply reads $\tau_{l} \approx 1.17 \tau_{\text {osc }}$.

We now turn to the characterization of the relevant timescale associated with the hole formation on smooth substrate. The latter is naturally measured as the time $\tau_{h}$ to nucleate a hole in the lamella, as reported in Fig. 3 for different drop radii and velocities. Overall, $\tau_{h}$ increases with $R_{0}$, and decreases with $V$, thus showing a velocity dependency unlike the previous drop impact typical time. As a consequence, if the impact velocity is below a critical value $V_{c}$, discussed hereafter, $\tau_{h}$ becomes larger than $\tau_{l}$, and therefore no hole is formed.

We observe that on a smooth surface, this critical velocity is always larger than the threshold velocity for classical splash, which then appears as the relevant mechanism for drop fragmentation on defect-free surfaces. However, the

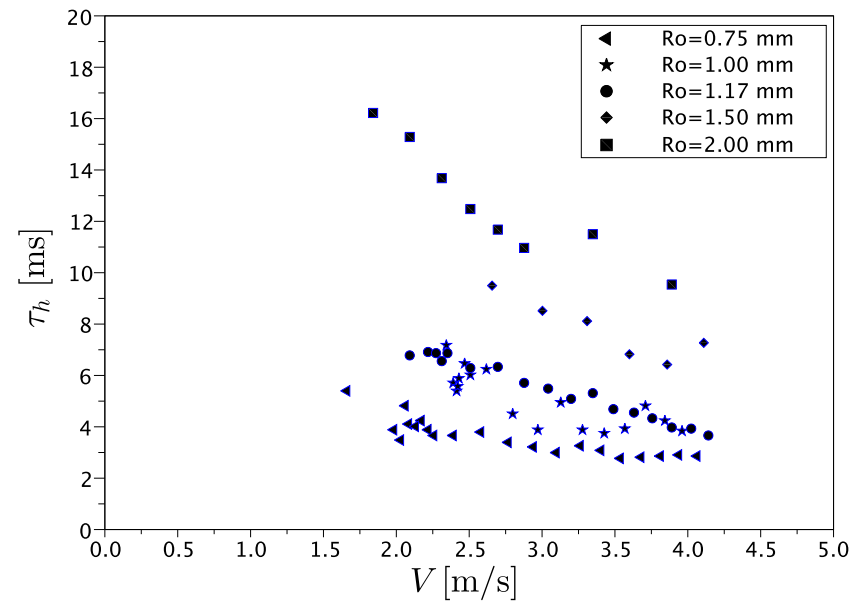

FIG. 3. (Color online) Impact on a smooth substrate. Time $\tau_{h}$ to nucleate a hole vs the drop velocity $V$ for several drop radii.

situation can be strongly modified on real surfaces with finite roughness. As shown below, the hole formation is indeed very sensitive to the presence of a microscopic defect which can trigger its appearance and shift it toward lower impact velocity. It thus makes the liquid lamella rupture by hole growth the relevant mechanism for satellite droplet formation.

Experimentally, a single model-defect is used to achieve quantitative insight into this process. It consists of a 30-300 $\mu \mathrm{m}$ spherical glass or borosilicate bead that is sintered onto a smooth silicon wafer.

More precisely, a defect of selected diameter is withdrawn from a bench of polydisperse beads dispersed onto a rough hydrophobic substrate with an atomic force microscope cantilever. Electrostatic and van der Waals interactions are restricted with the hydrophobic substrate intrinsically; therefore, the bead remains attached to the cantilever and is then gently dropped off on the smooth silicon wafer. After deposition, it is heated at $700{ }^{\circ} \mathrm{C}$ for $2 \mathrm{~h}$, allowing a smooth sintering. Bead size and circularity have been measured by profilometry and optical microscopy.

Now, as already stated, when a drop impacts near such a defect, the formation of a hole in the lamella happens earlier and at lower impact velocity (compared to defect-free substrate) and takes place on the defect, as shown in Fig. 1(d). Such hole formation at the defect could result from different possible mechanisms such as bubble entrapment at the defect, or nucleate boiling at the-presumably cooler-tip of the protruding defect. The important point here, however, is that in both cases the size of the defect fully determines the size of the induced hole. It acts as a puncturing object of set dimensions, thus imposing conditions on film characteristics for hole opening ${ }^{26}$ (see details hereafter).

As before, the typical timescale associated with the hole formation is characterized experimentally by measuring the elapsed time $\tau_{h}$ at which a hole in the lamella appears. The inset of Fig. 4 shows the measured $\tau_{h}$ as a function of the distance $x$ that separates the bead and the center of impact, for fixed defect and drop sizes, and impact velocity. This time appears essentially constant up to a distance of $0.5 R_{\max }$ 


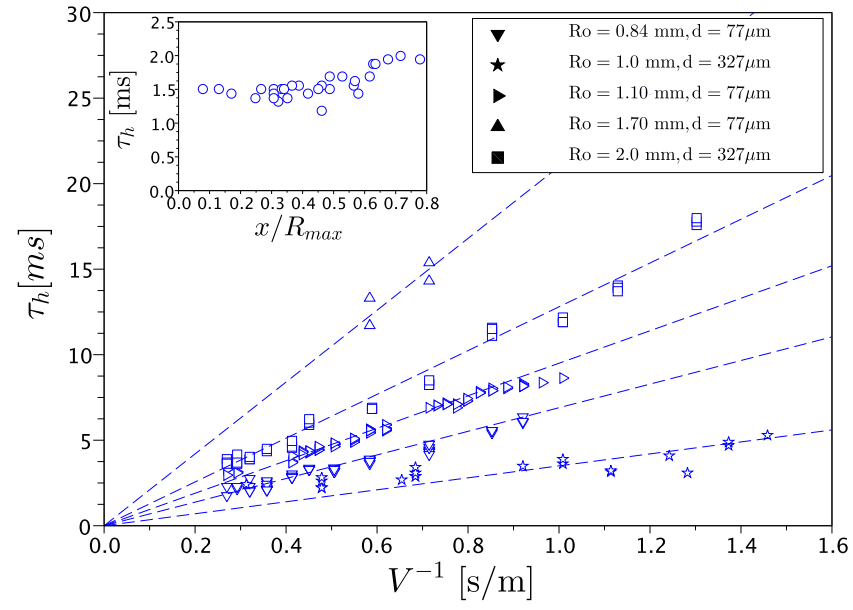

FIG. 4. (Color online) Time of formation of the hole $\tau_{h}$ after impact for several drop radii $R_{0}$ and defect diameters $d$ vs $V^{-1}$. Inset: time to nucleate a hole $\tau_{h}$ vs the radial coordinate $x$ for an impact velocity of $V=2.4 \mathrm{~m} / \mathrm{s}$, a drop size $R_{0}=1.46 \mathrm{~mm}$, and a defect size $d=290 \mu \mathrm{m}$.

and from now on, $\tau_{h}$ is taken as the value of this plateau. From there, we can concentrate on the measured values of the time $\tau_{h}$ for hole formation as a function of impact velocities $V$, drop radii $R_{0}$, and defect diameters $d$. Figure 4 reports this time $\tau_{h}$ versus $V^{-1}$ for several drop radii $R_{0}$ and defect diameters $d . \tau_{h}$ is a decreasing function of $V$ and $d$.

As for the timescale associated with drop impact, for which a scaling in $\tau_{\text {osc }}$ was evidenced, we now need to relate the time $\tau_{h}$ for hole formation to the different physical parameters of the problem. This requires additional description and characterization of the liquid sheet dynamical properties, a task for which the examination of the-subsequentgrowth of the lamella hole proved fruitful. The latter indeed expands due to surface tension and is found to remain nearly circular throughout expansion (aspect ratio between 1.0 and 1.1).

Two distinct parameters are then accessible experimentally: the variations of the hole radius with time, yielding a growth velocity $V_{h}=\mathrm{d} r / \mathrm{d} t$, and the drift velocity of the hole center. The latter is a consequence of the hole advection by the flow in the lamella along the radial coordinate. In the first approximation, we thus identify the center drift with the velocity $V_{l}$ of the liquid in the lamella. This drift velocity is found essentially constant during hole opening, and the deduced $V_{l}$ is reported in Fig. 5 versus the impact velocity. Overall, a linear dependency is observed. Hence, at this level of description, we retain that $V_{l} \sim V$.

Now, examining the hole growth dynamics, we show that the radius of the opening hole $r$ increases linearly with time, as reported in the inset of Fig. 6, in agreement with a constant dewetting velocity. This is reminiscent of the wellknown inertial dewetting of a liquid sheet of thickness $h,{ }^{27,28}$ whose front velocity writes $\sqrt{2 \gamma / \rho h}$. As already mentioned, we expect here the puncturing defect size to set the film characteristics $h$ of hole formation (opening). Indeed, plotting $V_{h}$ versus defect size $d$ in Fig. 6 shows a very good fit with $V_{h} \approx 2 \sqrt{2 \gamma / \rho d}$. This corresponds to a critical lamella thickness for a hole growth of $h=d / 4$. This is in full agree-

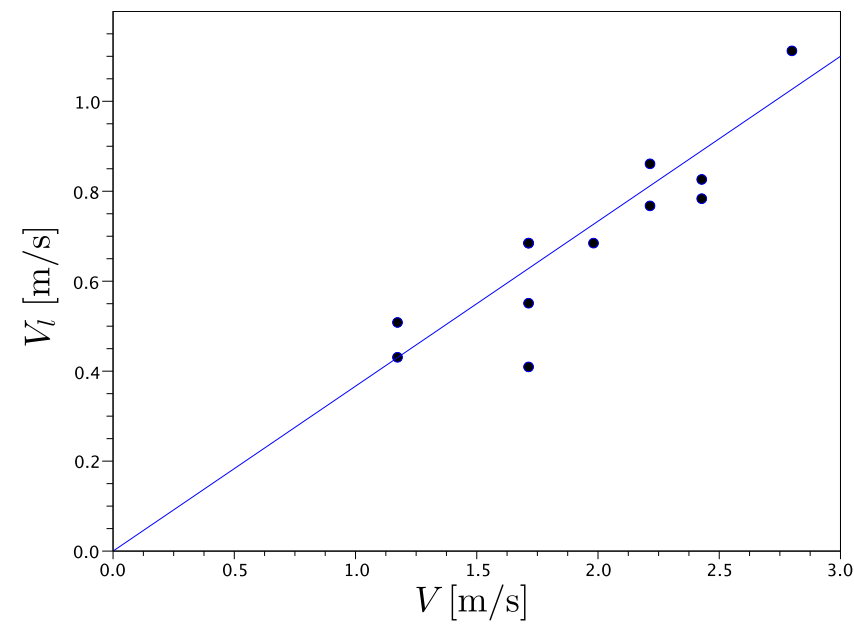

FIG. 5. (Color online) Velocity of the lamella $V_{l}$ vs impact velocity $\left(R_{0}=1.5 \mathrm{~mm}, d=77 \mu \mathrm{m}\right)$.

ment with the expected size threshold, in nonwetting situation, for hole expansion in a liquid lamella. ${ }^{26}$ Here, in practice, the hole created at the defect does not grow, until when the lamella has thinned down to the critical thickness.

As shown in the right inset of Fig. 6, the hole-opening velocity appears to be independent of the position of the defect $x$, which suggests that the lamella reaches a uniform thickness. If so, it is straightforward that, from the volume conservation in the expanding lamella, the fluid velocity should decrease with the radial position. Thus, it is clear that retaining a "mean" lamella velocity $V_{l} \sim V$, as presented above, only constitutes a first step modeling. A more detailed determination of $V_{l}$ with time and position is a route for future investigations beyond this global description.

Thus, at first approximation, here we assume a constant velocity in the lamella of uniform thickness. The radius of the slab increases with time as $R=V_{l} t$, if we do not take into account the rim. This approximation has already been shown

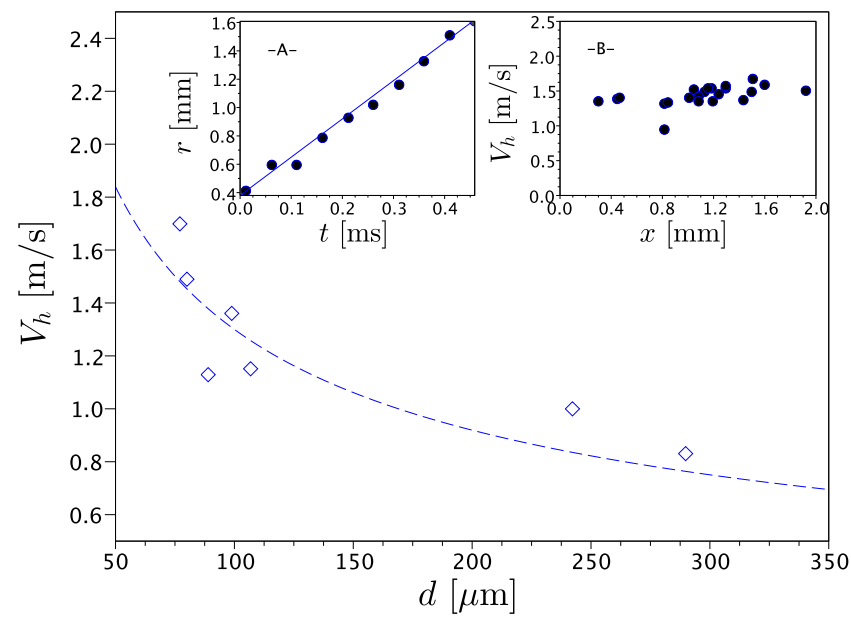

FIG. 6. (Color online) Dewetting velocity of the hole $V_{h}$ vs defect size $d$. The dashed blue line corresponds to the fit $V_{h}=2 \sqrt{2 \gamma / \rho d}$. Insets: (a) radius of a hole nucleated on a defect of size $d=290 \mu \mathrm{m}, R_{0}=1.46 \mathrm{~mm}$; (b) opening velocity of the hole $V_{h}$ on a defect vs the position of the defect $x$ $\left(d=107 \mu \mathrm{m}, R_{0}=1.46 \mathrm{~mm}\right)$. 


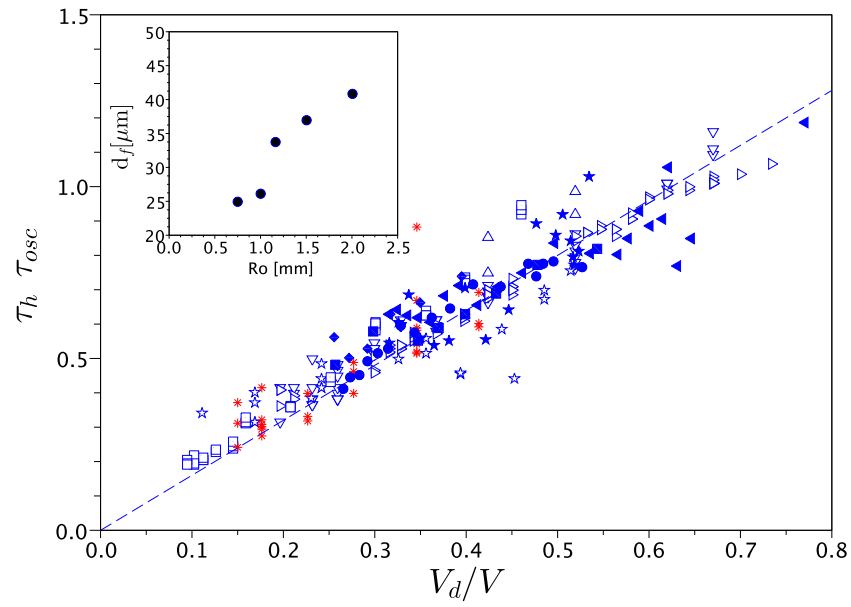

FIG. 7. (Color online) Comparison between $\tau_{h} / \tau_{\max }$ and $V_{d} / V$, and the results of the model (solid line). Inset: size of the equivalent defect $d_{f}$ vs $R_{0}$. Symbols as in Figs. 3 and 4. (*) corresponds to data obtained with acetone, $R_{0}=1.6 \mathrm{~mm}$.

successfully in the past. ${ }^{29}$ The global volume conservation reads $h \pi R^{2}=\frac{4}{3} \pi R_{0}^{3}$ or $h=4 R_{0}^{3} / 3 V_{l}^{2} t^{2}$. The hole will be formed for $h \approx d / 4$ after a time $\tau_{h}$,

$$
\tau_{h}=\sqrt{\frac{8}{3} \frac{2 R_{0}^{3}}{d}} \frac{1}{V_{l}} .
$$

With $V_{l} \sim V$,

$$
\tau_{h} / \tau_{\mathrm{osc}} \sim \frac{V_{d}}{V}
$$

where $V_{d}=\sqrt{2 \gamma / \rho d}$ is the dewetting velocity in a lamella of thickness $d$. The hole formation time versus $V^{-1}$ for several drop radii and defect sizes is reported in Fig. 4. A linear dependency with $1 / V$ is observed as expected from Eq. (4). Moreover, the lamella rupture time appears to be shorter when the defect size is increased. It indicates that the larger the defect, the earlier the formation of the hole. More quantitatively, Fig. 7 compares the scaled hole formation time $\tau_{h} / \tau_{\text {osc }}$ with the inverse of the scaled velocity $V_{d} / V$. The experimental data plotted in Fig. 4 collapse on the model curve defined by Eq. (4).

A criterion for hole formation on a defect of size $d$ can then be defined: the hole is formed if $\tau_{h}$ is smaller than the lifetime of the lamella $\tau_{l} \approx 1.17 \tau_{\text {osc }}$. This conditions allows us to define a critical impact velocity

$$
V_{c} \approx 1.4 \sqrt{\frac{2 \gamma}{\rho d}},
$$

which is independent of the drop radius $R_{0}$, and of the order of $0.9 \mathrm{~m} / \mathrm{s}$ for a $100 \mu \mathrm{m}$ defect for isopropanol drops.

Before discussing the importance of this hole-opening process for drop fragmentation, it is interesting to reconsider the smooth surface case within the present framework. Indeed, the data reported in Fig. 3 collapse well on the master curve of Fig. 7 if a fictive defect of size $d_{f}$ is introduced. The inset of Fig. 7 reports such $d_{f}$ values yielding best data collapse as a function of $R_{0}$. As can be seen, it increases almost linearly with $R_{0}$, in the range of our study, with $d_{f} \approx 30 \mu \mathrm{m}$ for a drop of radius $R_{0}=1 \mathrm{~mm}$. The origin of this length remains unclear. However, some experiments $^{13,14,16}$ already showed that a bubble, reported to have a size of $25 \mu \mathrm{m}$, can be trapped during the impact due to capillary wave convergence at the center of the thin film or defect in the substrate. Here, even if such a small bubble cannot be observed with our set-up, it could very well act as a defect entailing dry spot formation.

With these different elements, characterizing the hole formation and growth process, the relevancy of this mechanism for drop fragmentation-as compared to classical splash-can be raised. As discussed before, the critical splash velocity in our system is given by $V>\sqrt{\mathrm{We}_{c} \gamma / \rho R_{0}}$ with $\mathrm{We}_{c}=160$. If we compare it to the threshold velocity $V_{c}$ defined by Eq. (5), a critical aspect ratio to get hole formation without splash is set by

$$
\frac{R_{0}}{d} \approx 40
$$

With the development of nanodroplets and nanoprinting, this phenomenon gives a limit for surface roughness relevancy in the phenomenon at stake. For example, surface defects of 10 $\mathrm{nm}$ could induce hole formation during the impact of a $1 \mu \mathrm{m}$ droplet intrinsically.

In practice, the fragmentation is the consequence of the collision of the expanding dry spot edge (inner rim) with that surrounding the receding slab (outer rim). But it occurs only when the effect of the collision is maximized, i.e., when both rims have comparable momentum. Thus, the velocity criterion associated with the sole hole nucleation [Eq. (5)] does not necessarily warrant efficient fragmentation. Since the outer rim mass is always larger than the hole edge mass, the momentum is comparable when the outer rim is almost motionless. Thus, taking into account that the fragmentation would occur when the outer rim starts to recede, i.e., at $t \approx \tau_{\max }$, the critical velocity for drop fragmentation is better given by $V_{c}^{\text {frag }}=4 V_{d}$. This entails a critical aspect ratio of 5 , in better agreement with our observations [see Fig. 1(d)].

As a conclusion, a mechanism different from the usual splash can be responsible for the fragmentation of a drop impacting on a hot plate (i.e., in a nonwetting situation). For a smooth surface, the rupture spot appears at the center of the impact as if a micrometric defect $(30 \mu \mathrm{m}$ for a millimetric drop) of some sort was present on the surface. On the other hand, the hole formation can be triggered by a defect, which acts as a rupture spot. Moreover, the analysis of hole dynamics provides information on thickness and liquid flow velocity in the lamella for Leidenfrost impact.

Finally, the relevancy of such a mechanism compared to splash is discussed, and we show that this is the main mechanism of drop fragmentation, provided that defect and drop sizes are in a certain ratio. This work is a starting point in model conditions (a single defect in a nonwetting situation) to understand hole-induced drop fragmentation at impact, and should be generalized to various surface wettabilities and to multiple defects. The influence of a single defect on splash threshold must be studied in connection to observations made for patterned substrates. ${ }^{30}$ 
The authors thank C. Clanet and L. Bocquet for useful discussions, H. Ferret for technical help, L. Cousin for preliminary measurements, and A. Piednoir for bead deposition and sintering.

${ }^{1}$ D. B. van Dam and C. Le Clerc, "Experimental study of the impact of an ink-jet printed droplet on a solid substrate," Phys. Fluids 16, 3403 (2004).

${ }^{2}$ V. Bergeron, D. Bonn, J. Y. Martin, and L. Vovelle, "Controlling droplet deposition with polymer additives," Nature (London) 405, 772 (2000).

${ }^{3} \mathrm{~A}$. M. Worthington, "On the form assumed by drops of liquids falling vertically on horizontal plate," Proc. R. Soc. London 25, 261 (1876).

${ }^{4}$ A. L. Biance, C. Clanet, and D. Quere, "Leidenfrost drops," Phys. Fluids 15, 1632 (2003).

${ }^{5}$ A. L. Biance, "Gouttes inertielles: De la caléfaction à l'étalement," Ph.D. thesis, Université Paris 6, 2004.

${ }^{6}$ A. L. Yarin, "Drop impact dynamics: Splashing, spreading, receding, bouncing," Annu. Rev. Fluid Mech. 38, 159 (2006).

${ }^{7}$ P. Tsai, S. Pacheco, C. Pirat, L. Lefferts, and D. Lohse, "Drop impact upon micro- and nanostructured superhydrophobic surfaces," Langmuir $\mathbf{2 5}$, 12293 (2009).

${ }^{8}$ L. Xu, W. W. Zhang, and S. R. Nagel, "Drop splashing on a dry smooth surface," Phys. Rev. Lett. 94, 184505 (2005).

${ }^{9}$ C. Josserand, L. Lemoyne, R. Troeger, and S. Zaleski, "Droplet impact on a dry surface: Triggering the splash with a small obstacle," J. Fluid Mech. 524, 47 (2005).

${ }^{10} \mathrm{~L}$. Xu, "Liquid drop splashing on smooth, rough, and textured surfaces," Phys. Rev. E 75, 056316 (2007).

${ }^{11}$ R. E. Pepper, L. Courbin, and H. A. Stone, "Splashing on elastic membranes: The importance of early-time dynamics," Phys. Fluids 20, 082103 (2008).

${ }^{12}$ R. Dhiman and S. Chandra, "Rupture of radially spreading liquid films," Phys. Fluids 20, 092104 (2008).

${ }^{13}$ S. T. Thoroddsen, K. Takehara, and T. G. Etoh, "Dewetting at the centre of a drop impact," Mod. Phys. Lett. B 23, 361 (2009).

${ }^{14}$ S. Chandra and C. T. Avedisian, "On the collision of a droplet with a solid surface," Proc. R. Soc. London, Ser. A 432, 13 (1991).
${ }^{15}$ N. Z. Mehdizadeh and S. Chandra, "Boiling during high velocity impact of water droplets on a hot stainless steel surface," Proc. R. Soc. London, Ser. A 462, 3115 (2006).

${ }^{16}$ R. Dhiman and S. Chandra, "Rupture of thin films during droplet impact," Proc. R. Soc. London, Ser. A 466, 1229 (2010).

${ }^{17}$ V. Vazquez, E. Alvarez, and J. M. Navaza, "Surface tension of alcohol +water from 20 to $50{ }^{\circ} \mathrm{C}$," J. Chem. Eng. Data 40, 611 (1995).

${ }^{18}$ A. L. Biance, F. Chevy, C. Clanet, G. Lagubeau, and D. Quere, "On the elasticity of an inertial liquid shock," J. Fluid Mech. 554, 47 (2006).

${ }^{19}$ I. V. Roisman, E. Berberovic, and C. Tropea, "Inertia dominated drop collisions. I. On the universal flow in the lamella," Phys. Fluids 21, 052103 (2009)

${ }^{20}$ J. Eggers, M. A. Fontelos, C. Josserand, and S. Zaleski, "Drop dynamics after impact on a solid wall: Theory and simulations," Phys. Fluids 22, 062101 (2010).

${ }^{21}$ L. H. Wachters and N. A. Westerling, "Heat transfer from a hot wall to impinging water drops in spheroidal state," Chem. Eng. Sci. 21, 1047 (1966).

${ }^{22}$ M. Bussmann, S. Chandra, and J. Mostaghimi, "Modeling the splash of a droplet impacting a solid surface," Phys. Fluids 12, 3121 (2000).

${ }^{23} \mathrm{~K}$. Range and F. Feuillebois, "Influence of surface roughness on liquid drop impact," J. Colloid Interface Sci. 203, 16 (1998).

${ }^{24}$ D. Richard, C. Clanet, and D. Quere, "Surface phenomena-Contact time of a bouncing drop," Nature (London) 417, 811 (2002).

${ }^{25}$ K. Okumura, F. Chevy, D. Richard, D. Quere, and C. Clanet, "Water spring: A model for bouncing drops," EPL 62, 237 (2003).

${ }^{26}$ G. I. Taylor and D. H. Michael, "Making holes in a sheet of fluid," J. Fluid Mech. 58, 625 (1973).

${ }^{27}$ G. I. Taylor, "The dynamics of thin sheets of fluid. 3. Disintegration of fluid sheets," Proc. R. Soc. London, Ser. A 253, 313 (1959).

${ }^{28}$ F. Culick, "Comments on a ruptured soap film," J. Appl. Phys. 31, 1128 (1960).

${ }^{29}$ C. Clanet, C. Beguin, D. Richard, and D. Quere, "Maximal deformation of an impacting drop," J. Fluid Mech. 517, 199 (2004).

${ }^{30}$ M. Reyssat, D. Richard, C. Clanet, and D. Quéré, "Dynamical superhydrophobicity," Faraday Discuss. 146, 19 (2010). 